\title{
Large Eddy Simulation of Supersonic Combustion Using the Eulerian Stochastic Fields Method
}

\section{Y. P. Almeida ${ }^{1} \cdot$ S. Navarro-Martinez ${ }^{1}$}

Received: 19 February 2019 / Accepted: 18 July 2019 / Published online: 3 September 2019

(c) The Author(s) 2019

\begin{abstract}
The development of supersonic combustion engines (scramjet-type) presents several challenges. Numerical simulations of scramjet engine combustion have become an attractive alternative to experimental investigations. However, supersonic combustion simulations still have several challenges: such as adequate modelling of shock/boundary layer and turbulence/chemistry interactions. The present work presents two large-eddy simulation probability density function (LES-PDF) novel formulations developed for high-speed applications. One is a conservative joint-scalar approach, where the joint-probability for reactive scalars and energy is solved, while the other is a joint velocity-scalar. The LES-PDF transport equations are solved using the Eulerian stochastic fields technique implemented in a density-based compressible solver. The performance of the models is verified through the simulations of two and three-dimensional supersonic reacting mixing layers and compared against DNS data from the literature. The results show that the joint-scalar formulation is accurate and robust, while the joint velocity-scalar closures require further development.
\end{abstract}

Keywords Supersonic combustion · LES · PDF

\section{Introduction}

Hypersonic aircraft technology can be achieved by the use of air-breathing scramjet engines. In these engines combustion occurs at supersonic speeds, which involves complex physical process such as shock-boundary layer interaction and turbulence-chemistry interaction, which occur under a wide range of scales. Computational Fluid Dynamics (CFD) simulations of such phenomena are still challenging and few numerical model exists [1] that can be used in high-speed and reacting flows.

Probability Density Function (PDF) methods can provide an elegant closure to the highly non-linear source term [2] occurring in such flows. PDF turbulence models do not

S. Navarro-Martinez

s.navarro@imperial.ac.uk

1 Department of Mechanical Engineering, Imperial College London, Exhibition Road,

South Kensington, London SW7 2AZ, UK 
require a very fine mesh, unlike quasi-linear approaches [3], and are therefore promising for turbulence-combustion modelling.

The first compressible PDF formulation was presented by Kollmann and co-workers [4, 5]. In their model the PDF description included variables, such as density, velocity, internal energy, mixture fraction and the relative rate of volume expansion. This representation allowed the fully closure of the reactive and convective terms. Nevertheless, probably due to its mathematical complexity, it was not been further explored.

Alternative compressible PDF models were developed by Delarue and Pope [6]. The authors presented a PDF formulation including velocity, internal energy, pressure and turbulence frequency. Nik et al. [7] expanded the model to account for chemical reactions. This model also provided fully closure for the reactive and convective terms in a Large Eddy Simulation (LES) framework. The presented PDF equation was then solved using a stochastic Lagrangian procedure, where Lagrangian (or notional) particles are tracked and their ensemble average represents the PDF. The process involved interpolation between the Eulerian mesh and the Lagrangian particles which lead to non-uniform spatial resolution of the PDF. To maintain uniform accuracy (and stability) additional approaches were introduced [8].

An alternative approach to the Lagrangian particles is the Eulerian formulation. In this method, first developed by Valiño [9] and Sabel'nikov and Soulard [10], the stochastic equations equivalent to the PDF are solved within a Eulerian framework. This system of equations can be easily implemented into Eulerian CFD solvers. The stochastic Eulerian approach has been used in compressible PDF simulations before [11], however, only mass fractions and enthalpy were considered and sub-grid pressure fluctuations were neglected. This approximation allowed the closure of the reactive term, as employed by [4-6]. This approach has also been commonly used by several authors [12-14] within the Lagrangian framework, however, its accuracy has not been quantitatively investigated yet.

This work investigates two new formulations based on a Eulerian compressible LESPDF modelling, recently published in [15]. One formulation is a joint scalar PDF model [11], employing a filtered pressure closure as suggested by [13], which does not require subgrid pressure fluctuations modelling. The other formulation is a joint velocity-scalar PDF model, resulting in a more complete approach as it can close both unresolved convective and source terms. Both LES-PDF approaches are solved using the Eulerian Monte-Carlo stochastic fields technique.

The performance of the models is investigated through two and three-dimensional supersonic reacting spatial mixing layers. Mixing layers show different degrees of mixing and turbulent strain, creating different combustion behaviour and therefore are prototype combustion benchmarks. The results are compared with predictions of the Smagorinsky model coupled with the quasi-laminar combustion (neglecting sub-grid fluctuations) and with literature DNS data [16].

\section{The LES-PDF Modelling}

\subsection{Joint scalar PDF (SPDF)}

The approach shown here has firstly been published in [15] and it is briefly revised. The joint scalar PDF model includes enthalpy and mass fractions variables into its sample space. The 
one-time one-point fine-grained Eulerian probability density function (PDF) is therefore defined as:

$$
f^{\prime}(\eta, Z ; x, t)=\delta(h(x, t)-\eta) \times \prod_{\alpha=1}^{N_{s}} \delta\left(Y_{\alpha}(x, t)-Z_{\alpha}\right)
$$

where $\eta, Z_{\alpha}$ are the sample enthalpy and mass fractions, $h(x, t)$ and $Y_{\alpha}(x, t)$ are the real enthalpy and mass fractions, respectively. A non-closed filtered transport equation for LESPDF can be obtained by deriving a transport equation for $f^{\prime}$ and applying a spatial filter to it [8]:

$$
\begin{aligned}
& \frac{\partial \bar{\rho} \tilde{f}}{\partial t}+\frac{\partial \bar{\rho} \tilde{u}_{i} \tilde{f}}{\partial x_{i}}=\frac{\partial}{\partial x_{i}}\left(\Gamma \frac{\partial \tilde{f}}{\partial x_{i}}\right)-\frac{\partial}{\partial Z_{\alpha}}\left(\bar{\rho} \widetilde{S_{\alpha} \mid \Psi} \tilde{f}\right)
\end{aligned}
$$

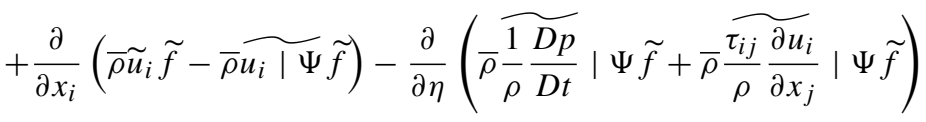

$$
\begin{aligned}
& -\frac{\partial^{2}}{\partial \psi_{\alpha} \partial \psi_{\beta}}\left(\bar{\rho} \Gamma \widehat{\frac{\partial \phi_{\alpha}}{\partial x_{i}} \frac{\partial \phi_{\beta}}{\partial x_{i}}} \mid \Psi \tilde{f}\right)
\end{aligned}
$$

where $(\widetilde{r})$ is the filtering operator and $\tilde{f}$ is the filtered PDF. The proposed LES-PDF equation relies on the assumption that the reactive source term is a function of sample variables and the filtered pressure. In this way, the reactive source term is partially modelled as $\widetilde{S}_{\alpha} \approx S_{\alpha}(\bar{p}, \Psi)$, where $\Psi=\left[\eta, Z_{\alpha}\right]$, in a similar fashion as [11, 13], although it is still accurate closed to regions of small compressibility or low-Mach number flows. The Smagorinsky model [17] is used to close the convective terms. The several remaining unclosed terms are modelled with the IEM micro-mixing model [18]. The joint scalar LES-PDF equation obtained is:

$$
\begin{aligned}
\frac{\partial \bar{\rho} \tilde{f}}{\partial t}+\frac{\partial \bar{\rho} \tilde{u}_{i} \tilde{f}}{\partial x_{i}}= & \frac{\partial}{\partial x_{i}}\left(\Gamma^{\prime} \frac{\partial \tilde{f}}{\partial x_{i}}\right)-\frac{\partial}{\partial Z_{\alpha}}\left(\bar{\rho} S_{\alpha}(\bar{p}, \Psi) \tilde{f}-\frac{1}{2} \frac{C_{Y_{\alpha}}}{\tau_{s g s}} \bar{\rho}\left(Z_{\alpha}-\tilde{Y}_{\alpha}\right) \tilde{f}\right) \\
& -\frac{\partial}{\partial \eta}\left(\frac{D \bar{p}}{D t} \tilde{f}+\tilde{\tau}_{i j} \frac{\partial \tilde{u}_{i}}{\partial x_{j}} \tilde{f}-\frac{1}{2} \frac{C_{H}}{\tau_{s g s}} \bar{\rho}(\eta-\tilde{h}) \tilde{f}\right)
\end{aligned}
$$

where $\Gamma^{\prime}=\mu / \sigma+\mu_{\text {sgs }} / \sigma_{\text {sgs }}$ is the total diffusion coefficient. A sub-grid mixing timescale is defined as in [19]:

$$
\tau_{s g s}=\left(\frac{\mu+\mu_{s g s}}{\bar{\rho} \Delta^{2}}\right)^{-1}\left(1-\exp \left(-\mathscr{R}^{2}\right)\right)
$$

where $\mathscr{R}$ is the ratio $\mu_{s g s} / \mu$ (akin to a sub-grid Reynolds number). The time-scale reverts to a molecular one when the local flow is laminar. The model constants $C_{Y_{\alpha}}$ and $C_{H}$ are equal to 2 following [20]. Equal diffusivity is assumed (unity Lewis number), where the Schmidt number $\sigma$ and Prandtl number are equal to unity, while their equivalent sub-grid number, $\sigma_{s g s}$ are equal to 0.7 . The conventional Smagorinsky model is employed to obtain the subgrid viscosity, $\mu_{s g s}$. The equations for the $\mathrm{n}^{\text {th }}$-set of Eulerian stochastic fields for the mass fractions and enthalpy can be obtained following [10]:

$$
\begin{aligned}
\frac{\partial \bar{\rho} \mathscr{Y}_{\alpha}^{n}}{\partial t}+\frac{\partial \bar{\rho} \tilde{u}_{i} \mathscr{Y}_{\alpha}^{n}}{\partial x_{i}}= & \frac{\partial}{\partial x_{i}}\left(\Gamma^{\prime} \frac{\partial \mathscr{Y}_{\alpha}^{n}}{\partial x_{i}}\right)+\bar{\rho} S_{\alpha}^{n}(\bar{p}, \Psi)-\frac{1}{2} \frac{C_{Y_{\alpha}}}{\tau_{s g s}} \bar{\rho}\left(\mathscr{Y}_{\alpha}^{n}-\tilde{Y}_{\alpha}\right) \\
& +\left(2 \bar{\rho} \Gamma^{\prime}\right)^{1 / 2} \frac{\partial \mathscr{Y}_{\alpha}^{n}}{\partial x_{i}} \frac{\mathrm{d} W_{i}^{n}}{\mathrm{~d} t}
\end{aligned}
$$




$$
\begin{aligned}
\frac{\partial \bar{\rho} \mathscr{H}^{n}}{\partial t}+\frac{\partial \bar{\rho} \tilde{u}_{i} \mathscr{H}^{n}}{\partial x_{i}}= & \frac{\partial}{\partial x_{i}}\left(\Gamma^{\prime} \frac{\partial \mathscr{H}^{n}}{\partial x_{i}}\right)+\frac{D \bar{p}}{D t}+\tilde{\tau}_{i j} \frac{\partial \tilde{u}_{i}}{\partial x_{j}}-\frac{1}{2} \frac{C_{H}}{\tau_{s g s}} \bar{\rho}\left(\mathscr{H}^{n}-\widetilde{h}\right) \\
& +\left(2 \bar{\rho} \Gamma^{\prime}\right)^{1 / 2} \frac{\partial \mathscr{H}^{n}}{\partial x_{i}} \frac{\mathrm{d} W_{i}^{n}}{\mathrm{~d} t}
\end{aligned}
$$

where $\mathscr{Y}_{\alpha}^{n}$ and $\mathscr{H}^{n}$ are the stochastic variables for mass fractions of the chemical specie $\alpha$ and enthalpy, respectively, of the stochastic field $n$. The Wiener process $d W_{i}^{n}$ is approximated by $d t^{1 / 2} \gamma$, where $\gamma=\{-1,1\}$ is a dichotomic vector, ensuring $\left\langle d W_{i}\right\rangle=0$. Filtered quantities are calculated from the average of the stochastic fields as $\widetilde{Q} \approx\langle Q\rangle$.

The filtered pressure field is therefore calculated, when applying the ideal gas law, without neglecting its sub-grid fluctuations terms:

$$
\bar{p}=\bar{\rho} \widetilde{R T} \approx \bar{\rho} R_{u}\left[\frac{1}{N_{F}} \sum_{n=1}^{N_{F}}\left(\sum_{\alpha=1}^{N_{s}} \frac{\mathscr{Y}_{\alpha}^{n}}{M W_{\alpha}}\right) T^{n}\right]
$$

where $R_{u}$ is the gas universal constant, $M W_{\alpha}$ is the molecular weight of the chemical specie $\alpha$ and $T^{n}$ is the temperature of the $n^{\text {th }}$ field. This approach has not been tried previously with the stochastic fields approach, only in Lagrangian formulations [13]. Accounting for sub-grid pressure fluctuations may improved accuracy in hydrocarbon fuels-pure oxygen systems [21].

\subsection{Joint velocity-scalar PDF (VSPDF)}

This new formulation, also published in [15], is a more complete approach. Here, the velocity components, along with density, mass fractions and total energy are included into the PDF sample space. This allows the exact closure of the convective and chemical source terms in the LES framework. The one-time one-point joint velocity-scalar fine-grained Eulerian PDF is defined as follows:

$$
\begin{aligned}
f^{\prime}(d, v, \zeta, Z ; x, t)= & \delta(\rho(x, t)-d) \prod_{i=1}^{3} \delta\left(u_{i}(x, t)-v_{i}\right) \\
& \times \delta\left(e_{t}(x, t)-\zeta\right) \prod_{\alpha=1}^{N_{s}} \delta\left(Y_{\alpha}(x, t)-Z_{\alpha}\right)
\end{aligned}
$$

where $d, v_{i}, \zeta$ and $Z_{\alpha}$ are the sample density, velocity, total energy and mass fraction, while $\rho(x, t), u_{i}(x, t), e_{t}(x, t)$ and $Y_{\alpha}(x, t)$ are the respective real fields. Following the same methods for the SPDF, it is possible to derive the following closed transport equation:

$$
\begin{aligned}
\frac{\partial \bar{\rho} \tilde{f}}{\partial t}+\frac{\partial \bar{\rho} v_{i} \tilde{f}}{\partial x_{i}}= & -\frac{\partial}{\partial d}\left(-\bar{\rho}^{2} \frac{\partial \tilde{u}_{i}}{\partial x_{i}} \tilde{f}\right) \\
& -\frac{\partial}{\partial Z_{\alpha}}\left(\frac{\partial \tilde{J}_{\alpha, i}}{\partial x_{i}} \tilde{f}+\bar{\rho} S_{\alpha}(\Phi) \tilde{f}-\frac{1}{2} C_{Y_{\alpha}} \frac{\epsilon}{k} \bar{\rho}\left(Z_{\alpha}-\tilde{Y}_{\alpha}\right) \tilde{f}\right) \\
& -\frac{\partial}{\partial v_{i}}\left(-\frac{\partial \bar{p}}{\partial x_{i}} \tilde{f}+\frac{\partial \tilde{\tau}_{i j}}{\partial x_{i}} \tilde{f}+\bar{\rho} G_{i j}\left(v_{j}-\tilde{u}_{j}\right) \tilde{f}\right) \\
& +\frac{\partial^{2}}{\partial v_{i} \partial v_{i}}\left(\frac{1}{2} C_{0} \epsilon_{s g s} \tilde{f}\right)-\frac{\partial}{\partial \zeta}\left(\frac{\partial \tilde{q}_{i}}{\partial x_{i}} \tilde{f}-\frac{\partial \bar{p} \tilde{u}_{i}}{\partial x_{i}} \tilde{f}+\frac{\partial \tilde{\tau}_{i j} \tilde{u}_{j}}{\partial x_{i}} \tilde{f}\right. \\
& \left.-\frac{1}{2} C_{e_{t}} \frac{\epsilon_{s g s}}{k_{s g s}} \bar{\rho}\left(\zeta-\tilde{e}_{t}\right) \tilde{f}\right)
\end{aligned}
$$


Table 1 Mass fractions at the inlet [16]

\begin{tabular}{lll}
\hline Specie & Fuel & Oxidizer \\
\hline$Y_{\mathrm{H}_{2}}$ & 0.05 & 0 \\
$Y_{\mathrm{O}_{2}}$ & 0 & 0.278 \\
$Y_{\mathrm{H}_{2} O}$ & 0 & 0.17 \\
$Y_{\mathrm{H}}$ & 0 & $5.60 \times 10^{-7}$ \\
$Y_{\mathrm{O}}$ & 0 & $1.55 \times 10^{-4}$ \\
$Y_{\mathrm{OH}}$ & 0 & $1.83 \times 10^{-3}$ \\
$Y_{\mathrm{HO}}$ & 0 & $5.10 \times 10^{-6}$ \\
$Y_{\mathrm{H}_{2} O_{2}}$ & 0 & $2.50 \times 10^{-7}$ \\
$Y_{\mathrm{N}_{2}}$ & 0.95 & 0.55 \\
\hline
\end{tabular}

The simplified Langevin model [2] is used to close the unknown terms for the velocity and density part. The tensor $G_{i j}$ is then defined as:

$$
G_{i j}=-\frac{\epsilon_{s g s}}{k_{s g s}}\left(\frac{1}{2}+\frac{3}{4} C_{0}\right) \delta_{i j}
$$

where $k_{s g s}$ and $\epsilon_{s g s}$ are the sub-grid kinetic energy and its dissipation respectively. The IEM micro-mixing model [18] is also chosen to close the remaining unknown terms on the total energy and mass fractions part.

Two further approximations are necessary to derive the stochastic field equations. The first is to substitute the pressure terms by the stochastic pressure field, $\mathscr{P}(x, t)$, instead of the filtered pressure, $\bar{p}$, neglecting sub-grid pressure fluctuations. This results in a different momentum equation from the Burgers' equation, preventing the occurrence of numerical shocks. The stochastic pressure can be directly obtained from stochastic variables $\mathscr{P} \equiv$ $\mathscr{P}\left(\varrho, \mathscr{U}_{i}, \mathscr{E}_{t}, \mathscr{Y}_{\alpha}\right)$ and present the same properties of the real pressure field.

The other approximation used is to neglect the stochastic difference on the right hand side of the continuity equation if written on conservative form, so it can ensure mass conservation for all set of stochastic fields. This approximation does not affect the first-moments and is exact if the density is constant. Although this approximation can be avoided in a Lagrangian framework, the SPDEs are aimed to be coupled with a Eulerian solver. This numerical approximation act as an additional force to prevent the fields from diverge striongly by adding or removing stochastic density. The proposed set of Eulerian SPDEs is therefore:

$$
\begin{gathered}
\frac{\partial \varrho^{n}}{\partial t}+\frac{\partial \varrho^{n} \mathscr{U}_{i}^{n}}{\partial x_{i}}=0 \\
\frac{\partial \varrho^{n} \mathscr{U}_{i}^{n}}{\partial t}+\frac{\partial \varrho^{n} \mathscr{U}_{j}^{n} \mathscr{U}_{i}^{n}}{\partial x_{j}}=-\frac{\partial \mathscr{P}^{n}}{\partial x_{i}}+\frac{\varrho^{n}}{\bar{\rho}} \frac{\partial \widetilde{\tau}_{i j}}{\partial x_{i}}+\varrho^{n} G_{i j}\left(\mathscr{U}_{j}^{n}-\widetilde{u}_{j}\right)+\varrho^{n} \sqrt{C_{0} \frac{\epsilon_{s g s}}{\bar{\rho}} \frac{\mathrm{d} W_{i}^{n}}{\mathrm{~d} t}} \\
\frac{\partial \varrho^{n} \mathscr{Y}_{\alpha}^{n}}{\partial t}+\frac{\partial \varrho^{n} \mathscr{U}_{i}^{n} \mathscr{Y}_{\alpha}^{n}}{\partial x_{i}}=\frac{\varrho^{n}}{\bar{\rho}} \frac{\partial \widetilde{J}_{\alpha, i}}{\partial x_{i}}+\varrho^{n} S_{\alpha}(\Psi)-\frac{1}{2} C_{Y_{\alpha}} \frac{\epsilon_{s g s}}{k_{s g s}} \varrho^{n}\left(\mathscr{Y}_{\alpha}^{n}-\widetilde{Y}_{\alpha}\right) \\
\frac{\partial \varrho^{n} \mathscr{E}_{t}^{n}}{\partial t}+\frac{\partial \varrho^{n} \mathscr{U}_{i}^{n} \mathscr{E}_{t}^{n}}{\partial x_{i}}=\frac{\varrho^{n}}{\bar{\rho}} \frac{\partial \widetilde{q}_{i}}{\partial x_{i}}-\frac{\varrho^{n}}{\bar{\rho}} \frac{\partial \bar{p} \tilde{u}_{i}}{\partial x_{i}}+\frac{\varrho^{n}}{\bar{\rho}} \frac{\partial \tilde{\tau}_{i j} \tilde{u}_{j}}{\partial x_{i}}-\frac{1}{2} C_{e_{t}} \frac{\epsilon_{s g s}}{k_{s g s}} \varrho^{n}\left(\mathscr{E}_{t} n-\tilde{e}_{t}\right)
\end{gathered}
$$

The employed closure relation for the dissipation of the sub-grid kinetic energy, $\epsilon_{s g s}$, is the following:

$$
\epsilon_{s g s}=C_{\epsilon} k_{s g s}^{3 / 2} / \Delta
$$




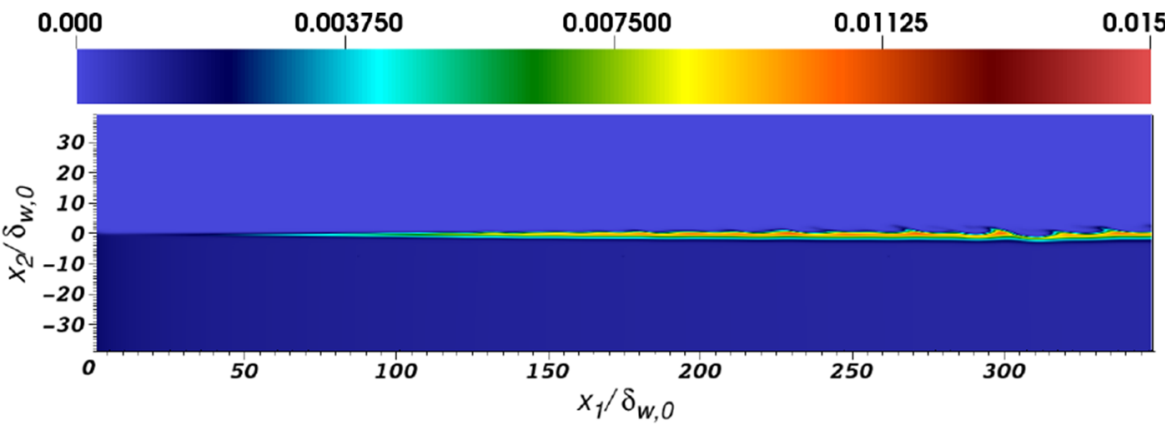

(a) Smagorinsky.

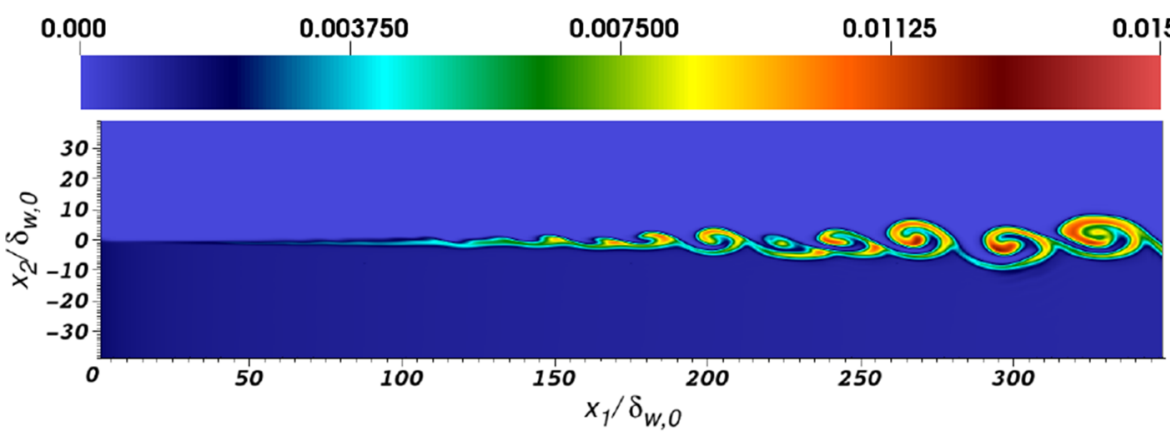

(b) SPDF.

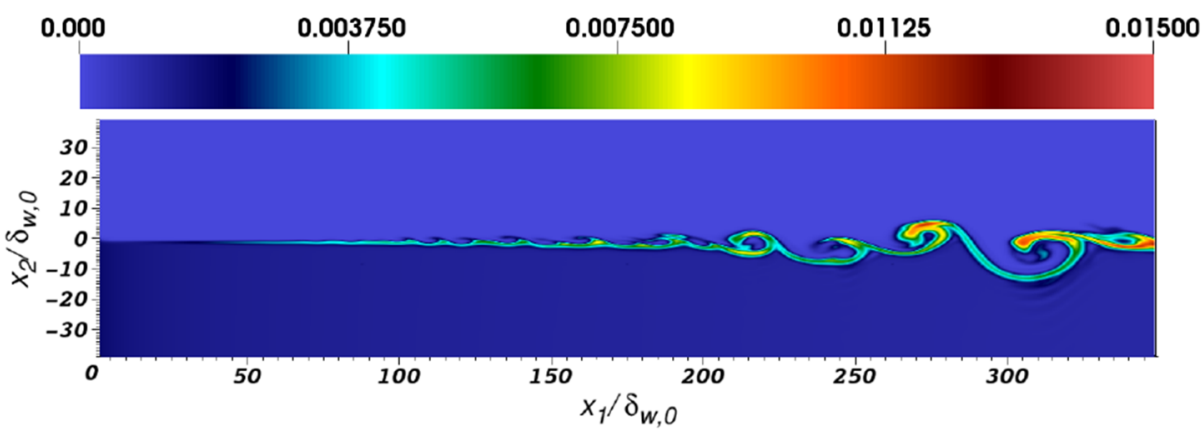

(c) VSPDF.

Fig. 1 Snapshot of the $\tilde{Y}_{O H}$ radical at $t=250 \mu \mathrm{s}$

where the constant $C_{\epsilon}$ is equal to 1.05 . The micro-mixing constants $C_{Y_{\alpha}}$ and $C_{e_{t}}$ are set to 2 and the Langevin constant is set to 2.1, following LES-PDF convention [8]. The sub-grid kinetic energy, $k_{s g s}$, can be directly obtained from the stochastic fields information:

$$
k_{s g s}=\frac{1}{2}\left(\frac{1}{N_{f}} \sum_{n=1}^{N_{f}}\left(\mathscr{U}_{i}^{n}-\tilde{u}_{i}\right)^{2}\right)
$$


At last, the filtered variables can be obtained from the average of the Eulerian stochastic fields. For a variable $Q(x, t)$, it is possible to recover the filtered and the Favre-filtered values:

$$
\bar{Q}=\frac{1}{N_{f}} \sum_{n=1}^{N_{f}} Q^{n} \quad \widetilde{Q}=\frac{\sum_{n=1}^{N_{f}} \varrho^{n} Q^{n}}{\sum_{n=1}^{N_{f}} \varrho^{n}}
$$

The developed Eulerian stochastic differential equations are equivalent to Eq. 9 with mild assumptions. The continuity and momentum equations resemble those of Azarnykh et al. [22]. This set of equations also does not generate the numerical shocks predicted in [23] if a conventional discretisation scheme is employed.

The novelty of the present VSPDF formulation stems from both the selection of independent variables and its solution method, which has advantages in high speed flows. The VSPDF approach includes a set of variables similar to Kollmann [5] but without a dilatation variable and with density instead of pressure, unlike the formulation of Nouri et al. [24]. This has benefits when combining the method with a density-based solver, which are typically used in high-speed flows. The use of Eulerian stochastic fields method to solve the VSPDF has not been used before for fully compressible and reactive flows. Moreover, the addition of an stochastic pressure, unlike other VPDF methods [6, 23] , allowed sub-grid pressure fluctuations as well as increase the stability of the SPDE system (11)-(14).

\section{Case Description}

Two and three-dimensional supersonic reactive mixing layers are simulated in order to investigate the new models performance. The configuration is the same presented by Ferrer [16]. The hot oxidizer stream $\left(\mathrm{N}_{2} / \mathrm{O}_{2} / \mathrm{H}_{2} \mathrm{O}\right)$ enters the domain at speed $U_{1}=1151.6$ $\mathrm{m} / \mathrm{s}(\mathrm{Ma}=1.5)$ and temperature $1475 \mathrm{~K}$ (hereafter stream 1$)$. The cold fuel stream $\left(\mathrm{N}_{2} / \mathrm{H}_{2}\right)$ inlet speed is $U_{2}=669.1 \mathrm{~m} / \mathrm{s}(\mathrm{Ma}=1.1)$ and temperature $545 \mathrm{~K}$ (stream 2$)$. The vorticity thickness is defined as:

$$
\delta_{w}=\frac{U_{1}-U_{2}}{\left|\partial \tilde{u}_{1} / \partial x_{2}\right|_{\max , 2}}
$$

where $|\cdot|_{\max , 2}$ indicates the maximum of the function at the crosswise coordinate $y$. The initial vorticity thickness is $\delta_{w, 0}=0.198 \mathrm{~mm}$. The composition of the streams are described in Table 1. The convective Mach number for this test case is 0.35 , resulting in mediumweak compressible effects. To trigger the instability growth, a perturbation is introduced in the crosswise component of the velocity [16] proportional to the convective velocity $U_{c}=\left(c_{1} U_{1}+c_{2} U_{2}\right) /\left(c_{1}+c_{2}\right)$. In the two dimensional case, a maximum perturbation of $0.1 U_{c}$ is used, while in the three-dimensional case the perturbation is periodic in the span-wise direction and its maximum size is $U_{c}$.

For the two-dimensional case a domain length of $L_{x}=350 \delta_{w, 0}$ and $L_{2}=80 \delta_{w, 0}$ is used. A mesh size of 36000 cells $(360 \times 108)$ is employed, using a stretching grid that increases the mesh elements density towards the centre line. For the three-dimensional simulation, the planar domain is extended to $L_{3}=40 \delta_{w, 0}$. The mesh size for all simulations is of 2.5 million $(360 \times 108 \times 64)$, also applying a stretching grid with more elements at the centreline. For the current LES, the filter width is approximately four times the Kolmogorov scale $\Delta \approx 4 \eta_{k}$, where $\eta_{k}$ is taken from the DNS [16] at $300 \delta_{w, 0}$ from the inlet. The mesh is relatively fine for a typical LES, with the filter width smaller that the Taylor micro-scale within the inertial sub-range. 


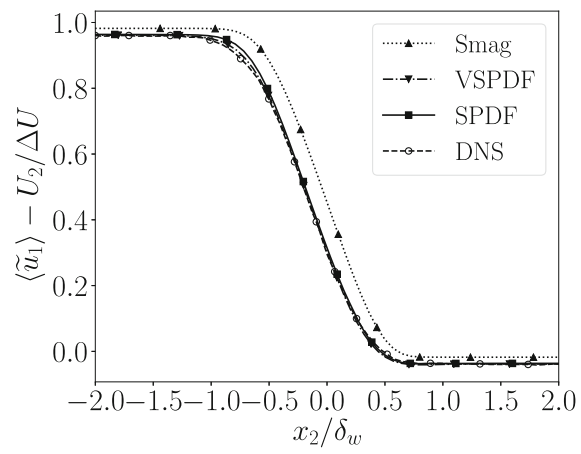

(a) $x_{1} / \delta_{w, 0}=150$

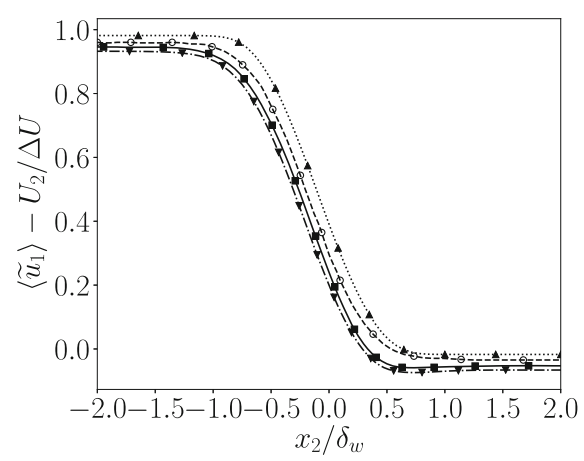

(c) $x_{1} / \delta_{w, 0}=250$

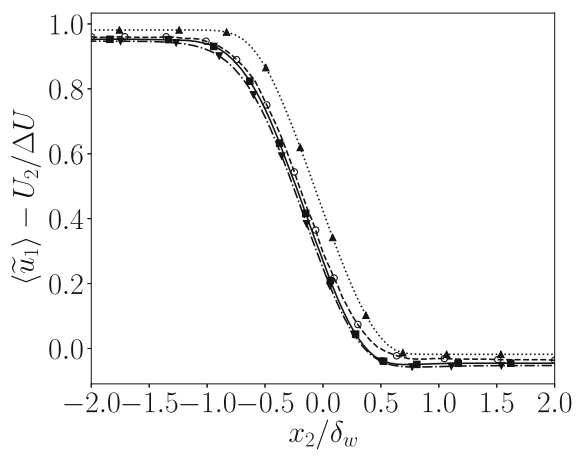

(b) $x_{1} / \delta_{w, 0}=200$

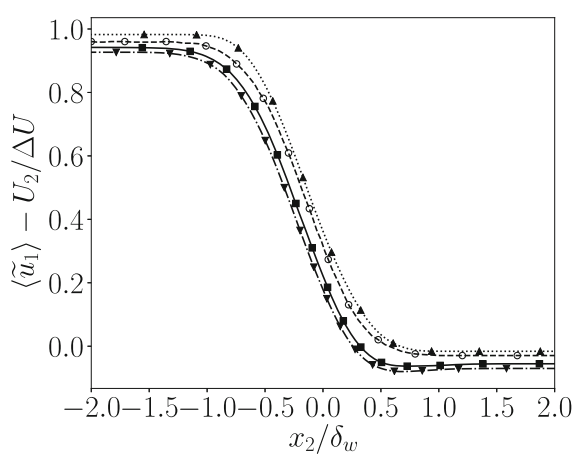

(d) $x_{1} / \delta_{w, 0}=300$

Fig. 2 Normalised time-averaged axial velocity

Simulations are carried out using the in-house code CompReal. First order zero-gradient boundary conditions are used at the outlet, top and bottom of the domain. Periodic boundary conditions are applied in the span-wise direction for the three-dimensional case. The $\mathrm{H}_{2} / \mathrm{O}_{2}$ skeletal mechanism of Yetteret al. [25] is used to describe the hydrogen combustion using 9 species and 19 reactions. At the inlet the flow is supersonic and the boundary conditions are prescribed. In the original DNS [16] the chemical mechanism of O'Conaire et al. [26] was employed. This mechanism has the same number species as Yetter's [25] but two more steps and has a wider pressure applicability (up to 87 bar). However, in the relatively low range of pressures in the present test-case, the difference between mechanisms is expected to be small and the slightly faster Yetter mechanism is therefore retained.

A hybrid DRP-HLLC numerical scheme is used, where the low-dispersive DRP scheme employs a 13 points stencil and fourth order accuracy in regions with small density/scalar gradients, and the HLLC-TVD second order is used in regions with sharp gradients. A fourth-order central differences discretization is used to obtain the remaining derivatives. All equations are integrated with an explicit third order Runge-Kutta scheme, whereas for the stochastic equations the order is reduced to strong 0.5 -order and weak first-order accuracy. A time step of $5.0 \times 10^{-9}$ secs is employed to ensure that the acoustic Courant number 


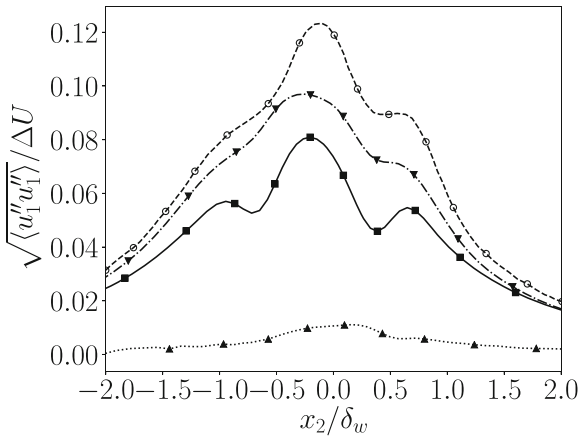

(a) $x_{1} / \delta_{w, 0}=150$

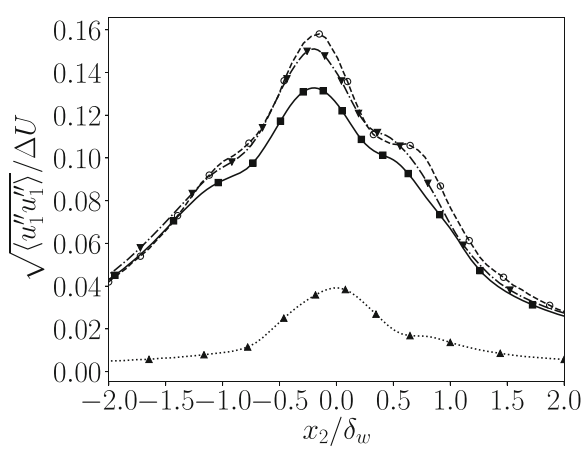

(c) $x_{1} / \delta_{w, 0}=250$

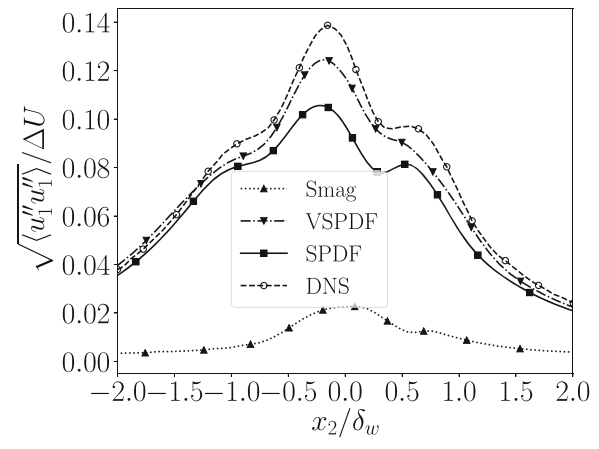

(b) $x_{1} / \delta_{w, 0}=200$

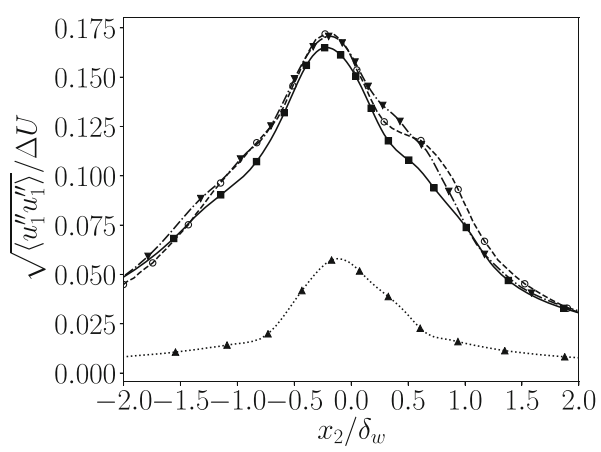

(d) $x_{1} / \delta_{w, 0}=300$

Fig. 3 Normalised time-averaged axial velocity fluctuation rms

does not increase above 0.2 for both cases. The simulations are performed using 8 stochastic fields in accord with other works in the literature [15, 19, 20, 27, 28]. The results are compared with the two and three-dimensional DNS results of Ferrer [16].

\section{Results}

\subsection{Two-dimensional case}

The two-dimensional mixing layer is not representative of real turbulence and therefore consequences for LES modelling should be handled with caution. Nevertheless, the proposed PDF models represent the unresolved contributions and therefore its relative behaviour can be compared. The instantaneous mass fraction of the radical $O H$ is displayed in Fig. 1, where the mixing layer growth and the combustion process are observed for the different models. The Smagorinsky model predicts a slow growth of the mixing layer, probably due to the large associated turbulence damping. The SPDF model generates adequate mixing levels and increases the growth of vorticity thickness both by increasing combustion level 


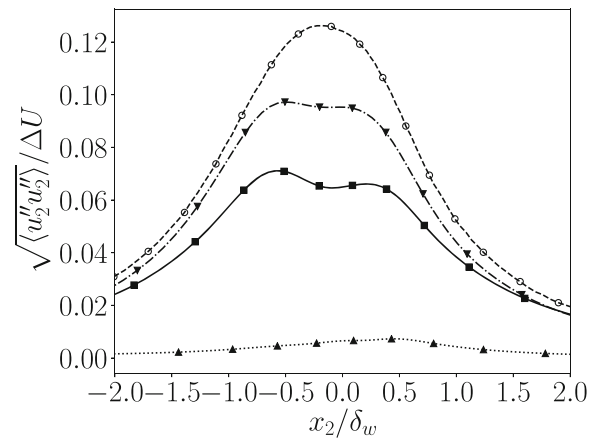

(a) $x_{1} / \delta_{w, 0}=150$

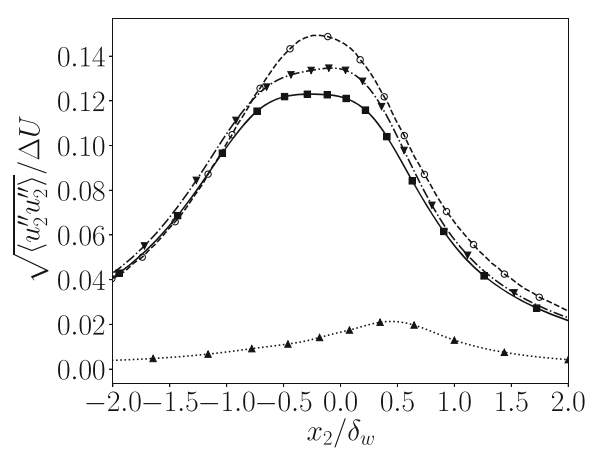

(c) $x_{1} / \delta_{w, 0}=250$

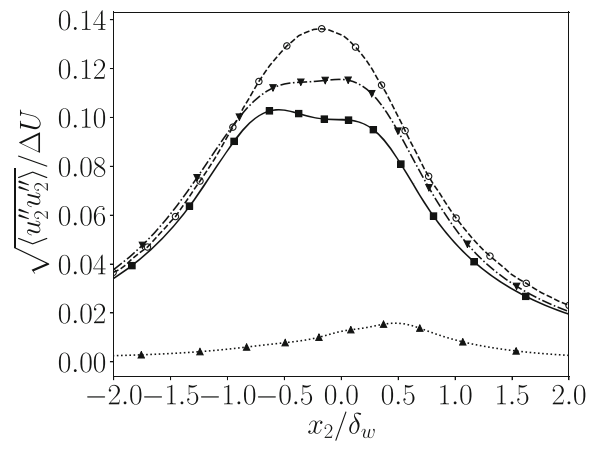

(b) $x_{1} / \delta_{w, 0}=200$

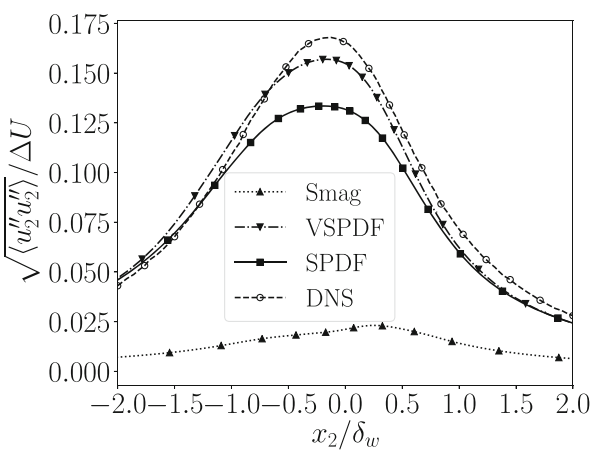

(d) $x_{1} / \delta_{w, 0}=300$

Fig. 4 Normalised time averaged crosswise velocity fluctuation rms

and the pressure correction in Eq. 7. The VSPDF formulation follows similar trend. However, stochastic noise can be observed at the end of the domain. The vortices in the VSPDF model are more distorted than the SPDF and the $O H$ net production is lower.

The averaged axial velocity at different downstream positions is shown in Fig. 2. The self-similarity of the velocity profiles is noticeable. Overall, SPDF and VSPDF results agree better with DNS data than Smagorinsky. Up to $x_{1} / \delta_{w, 0}=250$ the SPDF has a nonnegligible difference to the DNS results close to $x_{2} / \delta_{w}=0.5$ (close to the flame front), with the SPDF close second. In the last station, there is a difference between SPDF and VSPDF, which can be attributed to the stronger combustion of the SPDF approach. The Smagorinsky model presents very small momentum changes and remains mostly undisturbed throughout the domain. The traditional Smagorinsky model represents the convective part by adding sub-grid viscosity, which is not enough to simulate the mixing unless more points are used.

Figure 3 presents the normalised root mean square of the axial velocity fluctuation $u_{1}^{\prime \prime}$. The Smagorinsky model does not generate accurate results, with very low turbulent fluctuations. The SPDF and the VSPDF present good qualitative agreement with the DNS data. The VSPDF shows a close match with the DNS data at at $x_{1} / \delta_{w, 0}=250$ and $x_{1} / \delta_{w, 0}=300$. 


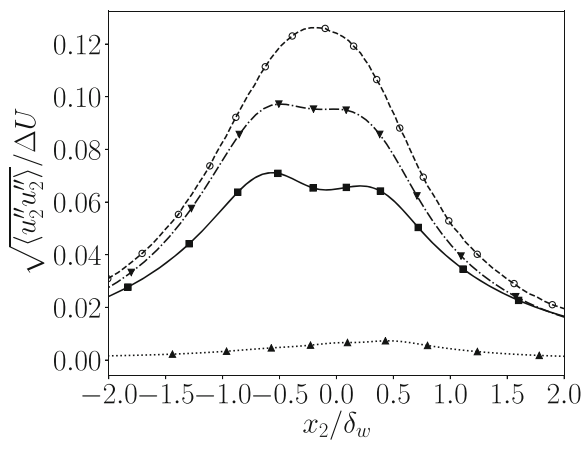

(a) $x_{1} / \delta_{w, 0}=150$

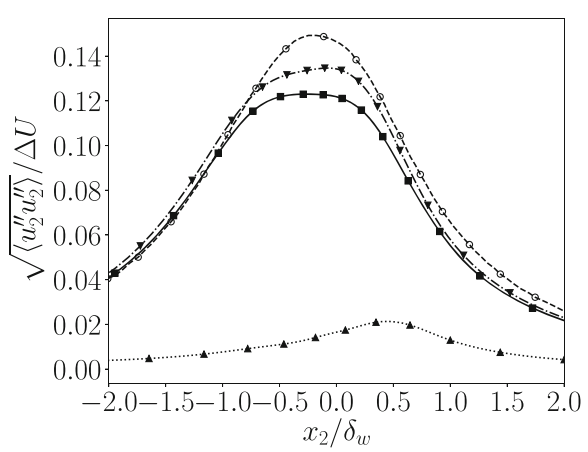

(c) $x_{1} / \delta_{w, 0}=250$

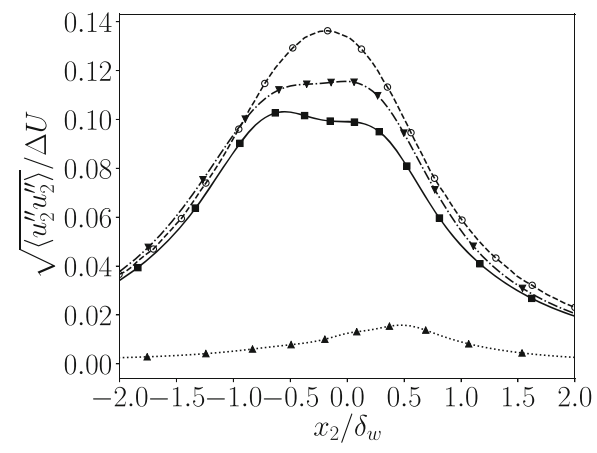

(b) $x_{1} / \delta_{w, 0}=200$

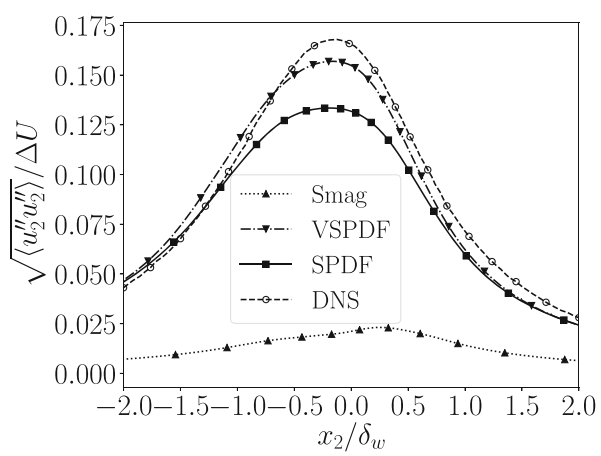

(d) $x_{1} / \delta_{w, 0}=300$

Fig. 5 Normalised time-averaged cross-correlation velocity fluctuation rms

Both PDF approaches show more than four times the amount of fluctuations than the simple Smagorinsky.

Similar behaviour is observed with the crosswise velocity fluctuations in Fig. 4, where PDF models agree well with the data downstream and the Smagorinsky model presents very small fluctuations.

Cross-correlations of the velocity components are shown in Fig. 5. Both models present good agreement with DNS data with the VSPDF showing slightly better results. Overall the velocity fluctuations are better represented by the VSPDF model, which provides an exact closure for the convective term. The Smagorinsky model suffers from the absence of an accurate combustion model and the required fine resolution to accurately reproduce the DNS velocity data.

Figure 6 presents the chemical species time-averaged profiles for the Smagorinsky, SPDF and VSPDF models (two-dimensional DNS species data not available [16] ). Both models enhance mixing level with larger spread of species than the Smagorinsky model. The $\mathrm{H}_{2}$ and $\mathrm{O}_{2}$ profiles of the SPDF and VSPDF models show similar trends, although the hydrogen has been more consumed in the SPDF model. The $\mathrm{H}_{2} \mathrm{O}$ profile (indicative of reaction progress) shows that the combustion on the Smagorinsky model still occurs but remains constrained to 


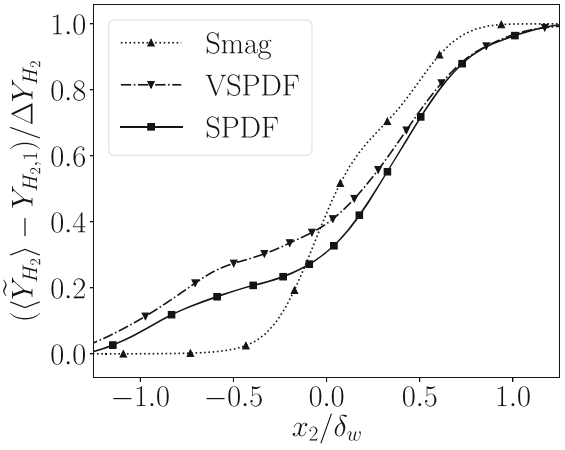

(a)

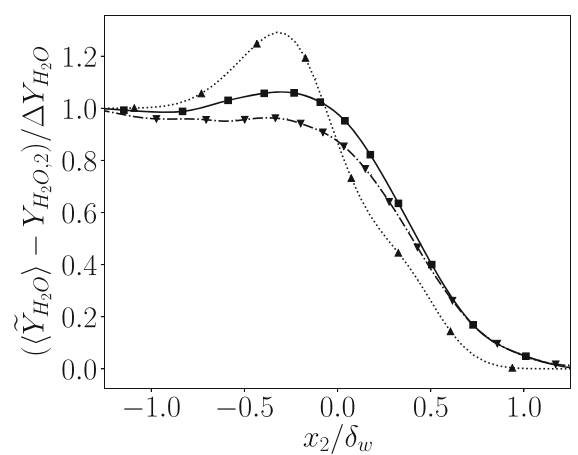

(c)

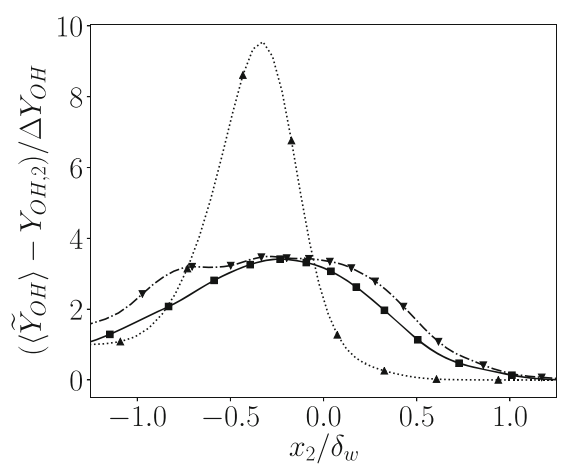

(e)

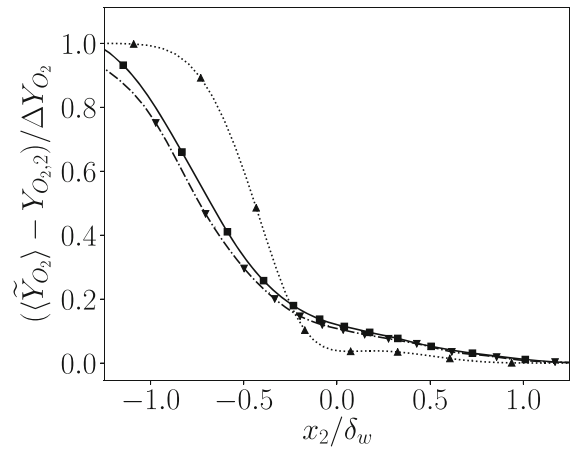

(b)

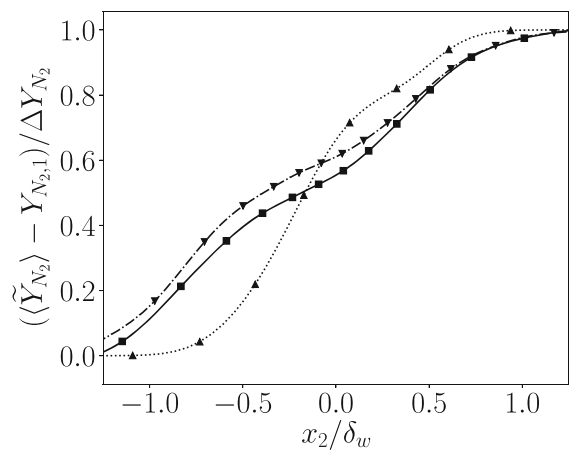

(d)

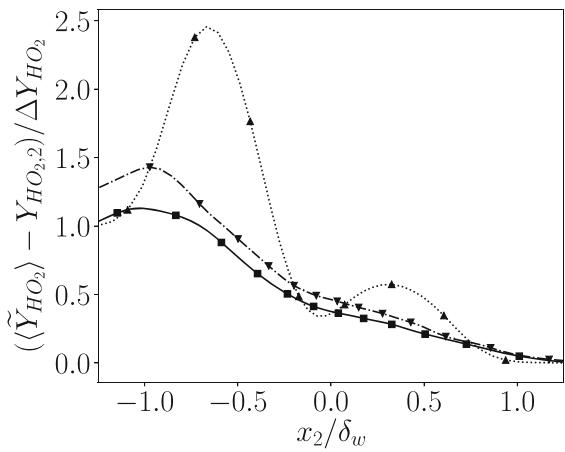

(f)

Fig. 6 Normalised time-averaged chemical species concentration at $x_{1} / \delta_{w, 0}=300$

the centreline. The Smagorinsky predictions of the $\mathrm{HO}_{2}$ radical (indicative of pre-ignition) shows an unphysical double peak, characteristic of a flame front.

The two-dimensional reactive mixing layer showcases the ability of the stochastic models to simulate supersonic flows and use a complex chemical mechanism for the hydrogen 
Fig. 7 Instantaneous $\widetilde{Y}_{O H}$ distribution (only values above $Y_{O H, 1}$ shown). Spatial coordinates normalised by $\delta_{w, 0}$

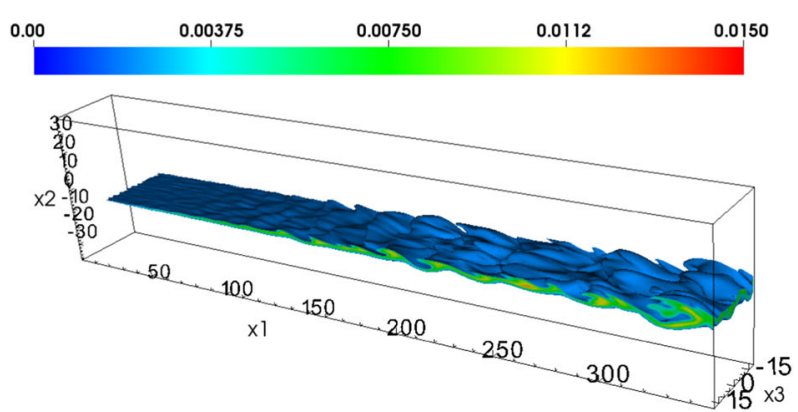

(a) Smagorinsky model.

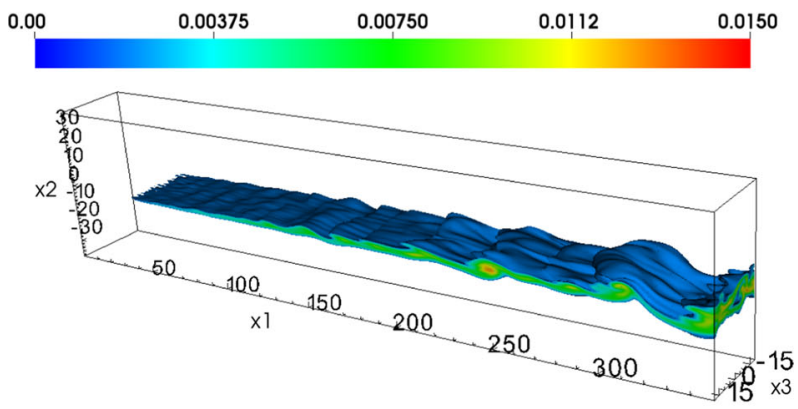

(b) SPDF model.

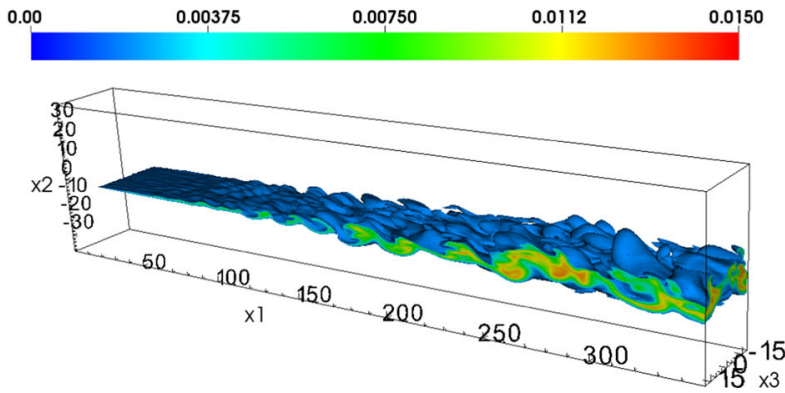

(c) VSPDF model.

combustion. Both PDF approaches show a good agreement with the DNS, while the Smagorinsky model does not generate the correct level of fluctuations.

\subsection{Three-dimensional case}

Figure 7 shows a snapshot of $O H$ mass-fraction distribution. Similar to the two-dimensional case, the comparison to the Smagorinsky model is performed as well. Unlike twodimensional, the Smagorinsky models shows a "turbulent" structure. The SPDF approach presents a larger defined vortical growth than Smagorinsky, while the VSPDF model has the earliest transition to turbulence and the highest $O H$ concentration (unlike the two-dimensional case). 


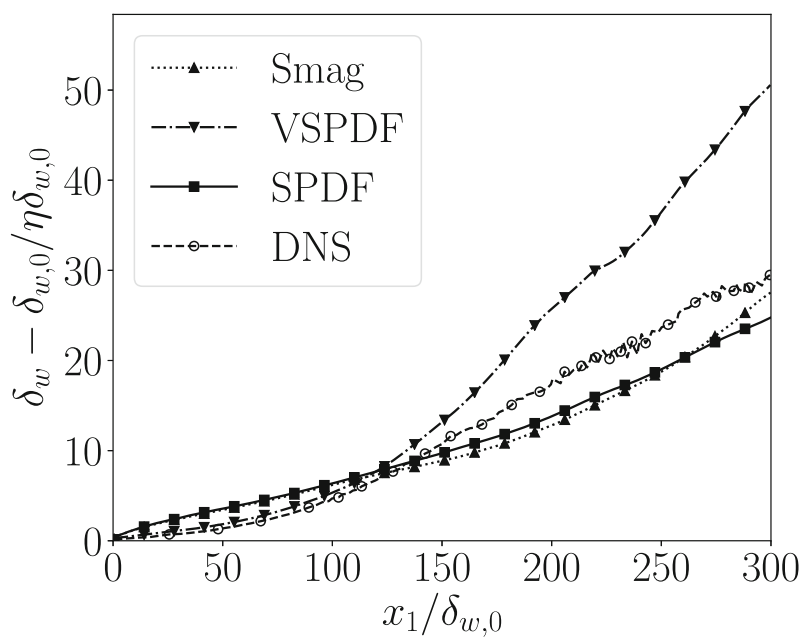

Fig. 8 Vorticity thickness growth

The vorticity thickness growth is presented in Fig. 8, comparing the DNS [16] and present LES approaches. The SPDF and the Smagorinsky models grow at a similar rate until approximately $x_{1} / \delta_{w, 0}=150$, when combustion occurs the results diverge. The SPDF and Smagorinsky follow closely to the DNS data. The VSPDF's growth rate is well above the DNS value from $x_{1} / \delta_{w, 0}=125$ onwards. This suggests that the VSPDF sub-grid modelling introduces excessive momentum mixing, which is not damped by the Langevin or micro-mixing.

Figure 9 presents the averaged axial velocity at $x_{1} / \delta_{w, 0}=300$ together with experimental data for non-reacting cases. As expected, the reacting cases reduce the axial-momentum due to transverse dilatation. The Smagorinsky model has the higher velocity at the end of the domain, mostly because of the delay in the overall combustion rate, and both the SPDF and

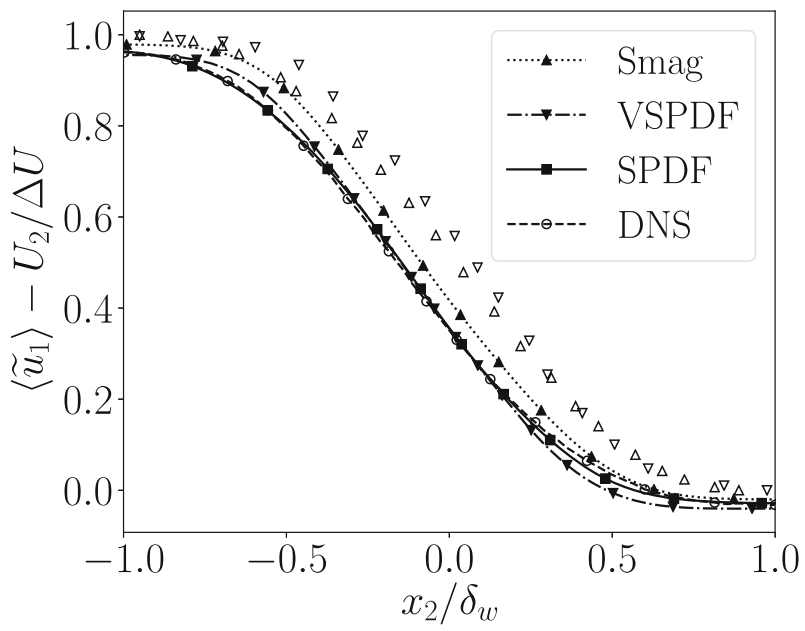

Fig. 9 Normalised averaged axial velocity at $x_{1} / \delta_{w, 0}=300$. Experimental data for non-reacting mixing layer of $[29](\triangle)$ and $[30](\nabla)$ 


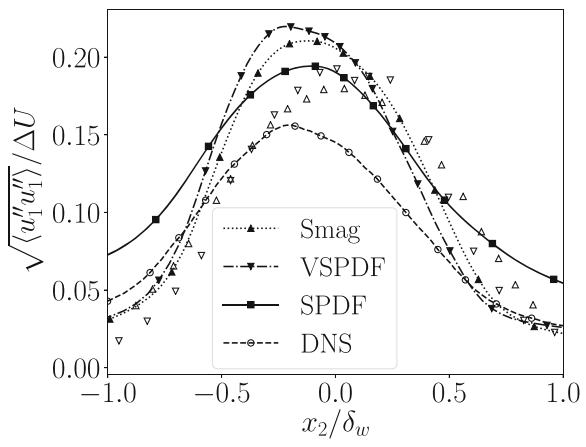

(a)

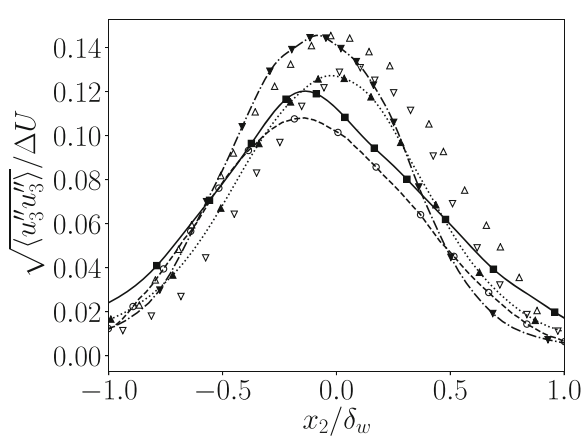

(c)

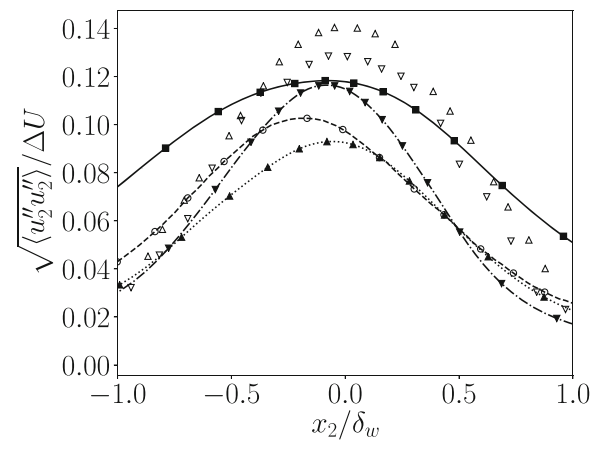

(b)

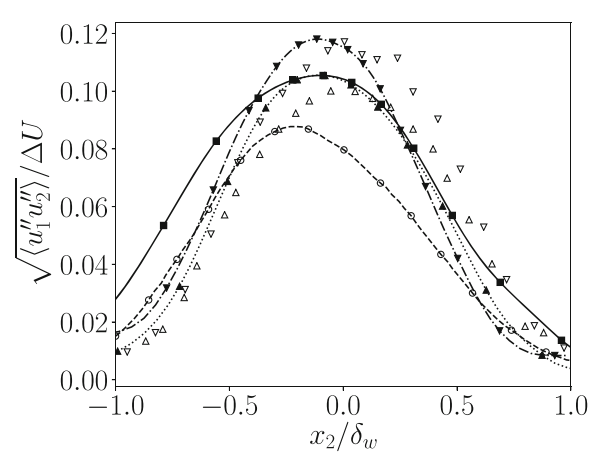

(d)

Fig. 10 Normalised averaged velocity $\mathrm{rms}$ at $x_{1} / \delta_{w, 0}=300$. Experimental data for non-reacting mixing layer of $[29](\triangle)$ and $[30](\nabla)$

VSPDF results follow closely the DNS data. It is worth noting that the SPDF model uses the same sub-grid closure for the convective term as quasi-laminar Smagorinsky, but captures better the momentum statistics through improvement of the filtered pressure coupling and the representation of sub-grid combustion processes.

Figure 10 shows the normalised averaged velocity $\mathrm{rms}$ at $x_{1} / \delta_{w, 0}=300$. The Smagorinsky model generates considerably better results compared to its two-dimensional counterpart (see Fig. 3). All models show reasonable qualitative agreement with the DNS data. The VSPDF generates higher fluctuation levels, closer to experimental data for nonreactive mixing layers, while the SPDF shows broader distribution of fluctuations, reaching beyond the vorticity thickness. DNS and model fluctuations levels are (in average) $10 \%$ below experimental non-reacting data, which indicates that the fluctuation levels are not strongly correlated to the combustion in the test case [16].

Chemical species mass fractions are shown in Figs. 11 (mean) and 12 (fluctuations). Major reactive species, $\mathrm{H}_{2}, \mathrm{O}_{2}$ and $\mathrm{H}_{2} \mathrm{O}$, are in good agreement with DNS results. The non-reactive species $N_{2}$ is a good indicator of the mixing levels, and although all models agree qualitatively with DNS, the SPDF approach diffuses more (also seen in major species profiles). Observing the $O H$ distribution, SPDF and VSPDF models generate a thicker flame than the DNS. The $\mathrm{HO}_{2}$ radical DNS profile could not be reproduced by any model. 


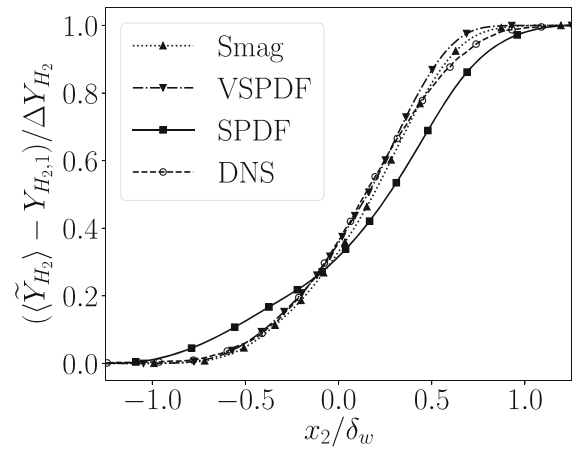

(a)

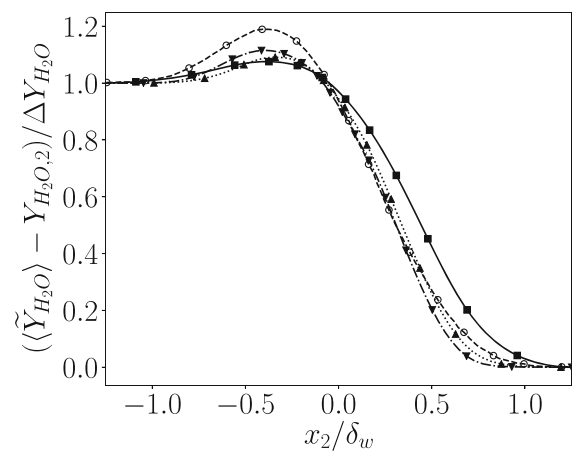

(c)

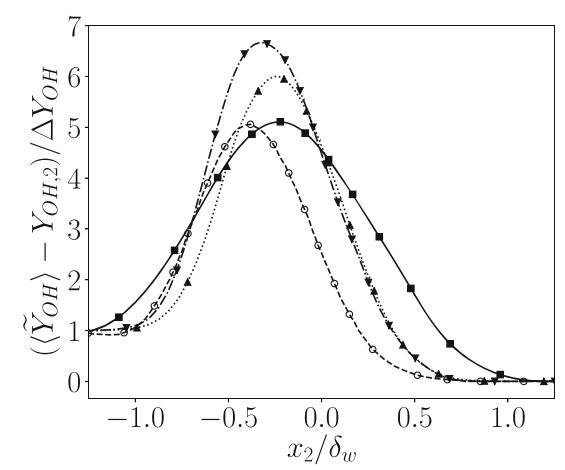

(e)

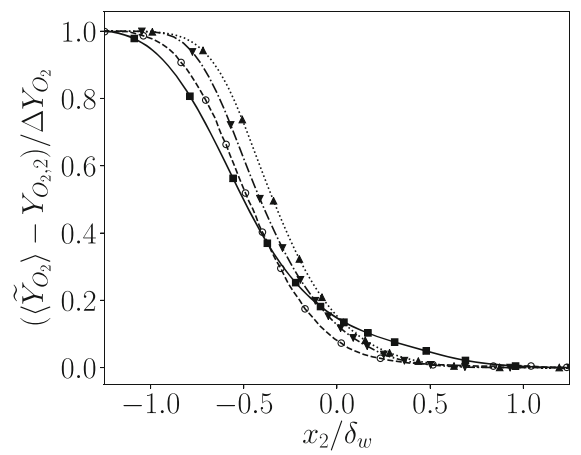

(b)

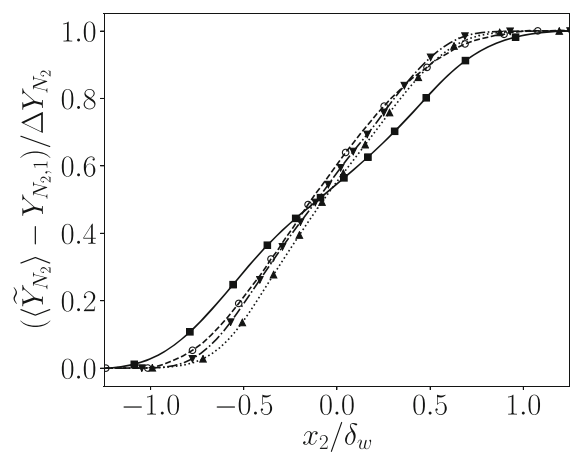

(d)

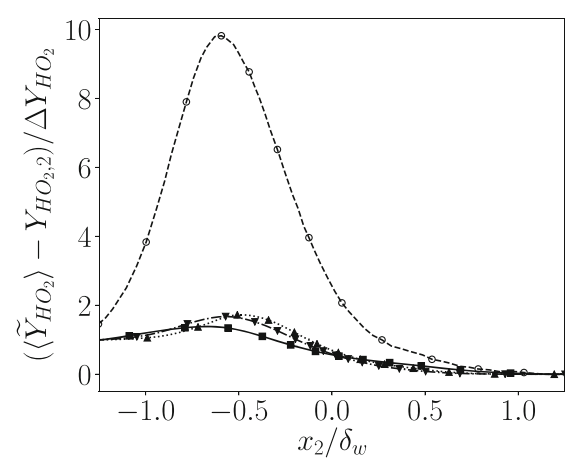

(f)

Fig. 11 Normalised chemical species mass fractions at $x_{1} / \delta_{w, 0}=300$

This may be attributed to the different chemical mechanism used in the simulations and DNS [26].

The mass fractions fluctuations profiles (Fig. 12) show similar behaviour. Predictions for $\mathrm{H}_{2}, \mathrm{O}_{2}, \mathrm{H}_{2} \mathrm{O}$ and $\mathrm{N}_{2}$ are higher for all approaches than the DNS values. However, with 


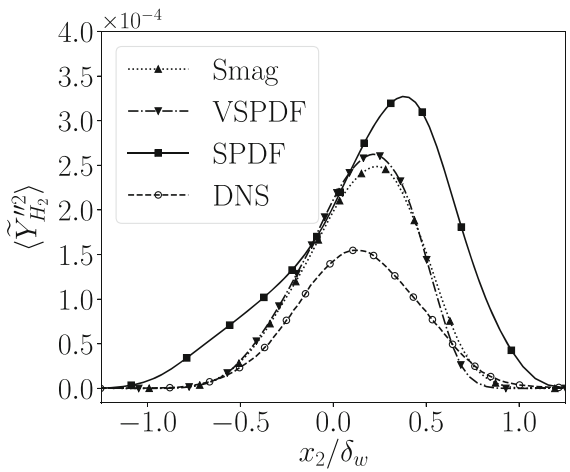

(a)

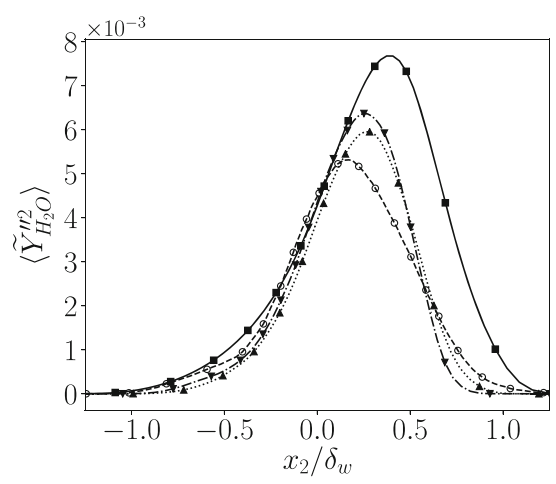

(c)

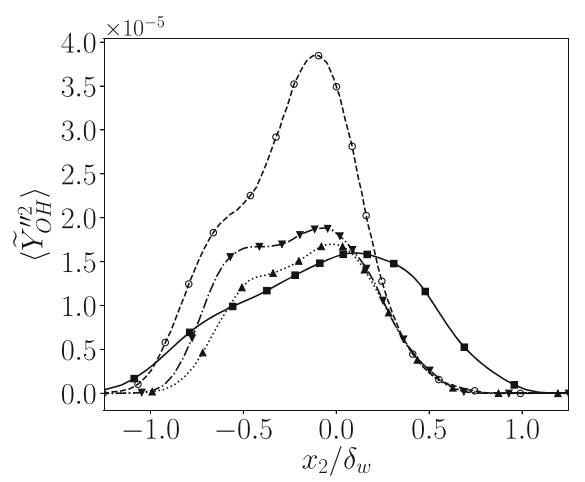

(e)

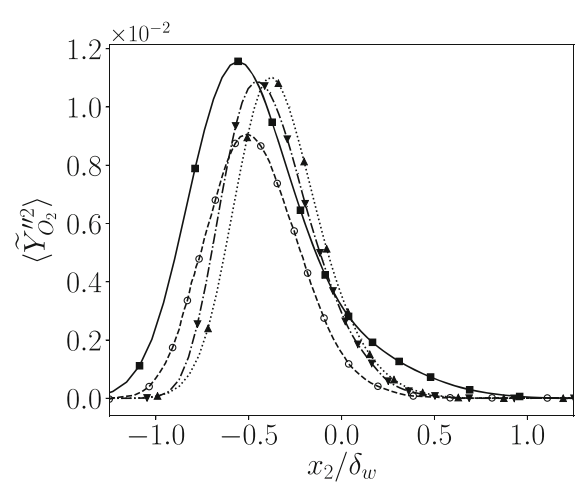

(b)

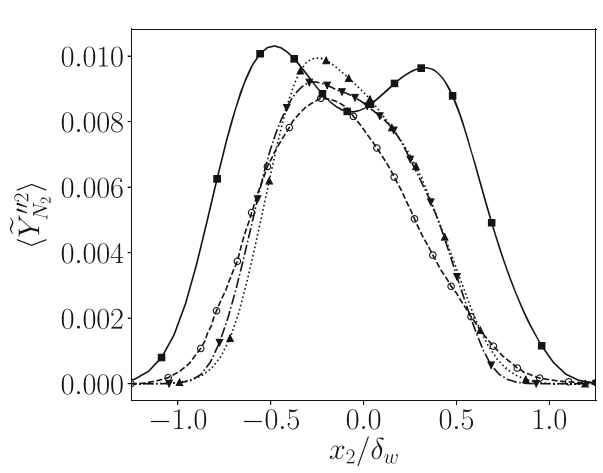

(d)

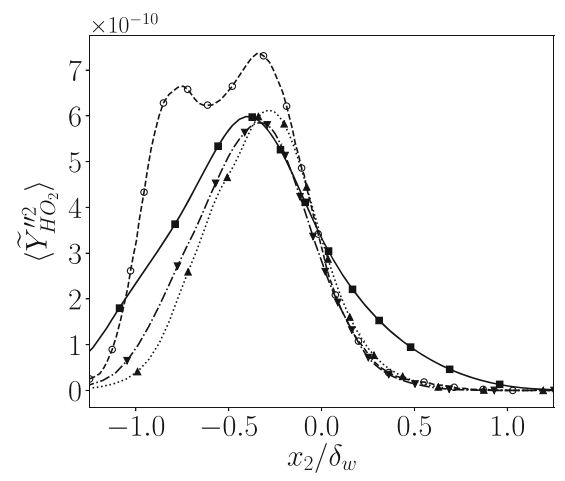

(f)

Fig. 12 Averaged chemical species mass fractions fluctuations at $x_{1} / \delta_{w, 0}=300$

good qualitative agreement. The SPDF model shows more diffusive behaviour overall, with the highest fluctuations. The SPDF $N_{2}$ fluctuation profile, presents two peaks, not seen in the DNS or in the other models. The $\mathrm{OH}$ and $\mathrm{HO}_{2}$ fluctuations are somewhat different from 
the DNS results, where the models predict lower fluctuation levels. The results, however, are of the same order of magnitude (even in the small $\mathrm{HO}_{2}$ ) and comparable to the DNS data.

\section{Conclusions}

Two and three-dimensional supersonic reactive mixing layer simulations have been performed using two new LES-PDF formulations using the Eulerian stochastic fields. The models have been compared with a conventional Smagorinsky approach with a quasilaminar treatment of the chemistry (neglecting sub-grid scalar fluctuations). All models use standard values for the sub-grid micro-mixing and Smagorinsky constants and no attempt has been done to calibrate or adjust the results to the DNS data.

The present SPDF model provides closure to the filtered pressure in the case of reactive ideal gases ensuring a strong coupling between the thermochemical states. The model improves the mixing predictions compared with conventional Smagorinsky model at the same resolution; even if both use the same sub-grid transport model. The SPDF approach show reasonable agreement with DNS data while the Smagorinsky model greatly underestimates the velocity fluctuations. This is probably due to the coupling of the pressure and the chemical state.

The VSPDF model increases the turbulence levels and at the same time reduce the amount of numerical diffusion in the scalars and $a$-priori maintains the closest coupling between thermodynamic and velocity. The results in the planar mixing layer are the closest to experimental data, however, it overestimates the growth in the vorticity layer in the three-dimensional simulation, compared to the SPDF or Smagorinsky approaches. These results suggest that the simplified Langevin model may need to account for sub-grid compressibility effects (for example following the modified model of [6] ). The present work complements the experimental validation in [15], using the benchmark configuration of a supersonic lifted burner [31], and shows that PDF approaches are a promising tool for supersonic combustion.

Acknowledgements Yuri P. Almeida acknowledges the financial support provided by the Brazilian National Council for Scientific and technological Development (CNPq) through the Science without Borders programme, grant no. 233815/2014-7.

Compliance with Ethical Standards This study was funded by the Brazilian National Council for Scientific and technological Development (CNPq) through the Science without Borders programme, grant no. $233815 / 2014-7$.

Conflict of interests The authors declare that they have no conflict of interest.

Open Access This article is distributed under the terms of the Creative Commons Attribution 4.0 International License (http://creativecommons.org/licenses/by/4.0/), which permits unrestricted use, distribution, and reproduction in any medium, provided you give appropriate credit to the original author(s) and the source, provide a link to the Creative Commons license, and indicate if changes were made.

\section{References}

1. Berglund, M., Fureby, C.: LES, of supersonic combustion in a scramjet engine model. Proc. Combust. Inst. 31(2), 2497-2504 (2007)

2. Pope, S.B.: PDF Methods for turbulent reactive flows. Prog. Energy Combust. Sci. 11, 119-192 (1985) 
3. Bouheraoua, L., Domingo, P., Ribert, G.: Large-eddy simulation of a supersonic lifted jet flame: Analysis of the turbulent flame base. Combust. Flame 179, 199-218 (2017)

4. Eifler, P., Kollmann, W.: AIAA 93-0448. PDF Prediction of Supersonic Hydrogen Flames. In: 31St Aerospace Sciences Meeting \& Exhibit. Reno (1993)

5. Kollmann, W.: A PDF closure for compressible turbulent chemically reacting flows. Tech. Rep. NAG 3-836 National Aeronautics and Space Administration - NASA (1992)

6. Delarue, B.J., Pope, S.B.: Application of PDF methods to compressible turbulent flows. Phys. Fluids 9(9), 2704-2715 (1997)

7. Nik, M.B., Givi, P., Madnia, C.K., Pope, S.B.: EPVS-FMDF for LES of High-Speed Turbulent Flows. In: 50Th AIAA Aerospace Sciences Meeting including the New Horizons Forum and Aerospace Exposition. Tennessee (2012)

8. Haworth, D.C.: Progress in probability density function methods for turbulent reacting flows. Prog. Energy Combust. Sci. 36, 168-259 (2010)

9. Valiño, L.: A Field Monte Carlo Formulation for Calculating the Probability Density Function of a Single Scalar in a Turbulent Flow. Flow, Turbul. Combust. 60, 157-172 (1998)

10. Sabel'nikov, V., Soulard, O.: Rapidly decorrelating velocity-field model as a tool for solving one-point Fokker-Planck equations for probability density functions of turbulent reactive scalars. Phys. Rev. E. 72, 1282-1284 (2005)

11. Gong, C., Jandi, M., Bai, X., Liang, J., Sun, M.: Large eddy simulation of hydrogen combustion in supersonic flows using an eulerian stochastic fields method. Int. J. Hydrogen Energy 42, 1264-1275 (2017)

12. Banaeizadeh, A., Li, Z., Jaberi, F.A.: compressible scalar filtered mass density function model for HighSpeed turbulent flows. AIAA J. 49(10), 2130-2143 (2011)

13. Gerlinger, P.: Lagrangian transported MDF methods for compressible high speed flows. J. Comput. Phys. 339, 68-95 (2017)

14. Möbus, H., Gerlinger, P., Brüggemann, D.: Scalar and joint scalar-velocity-frequency Monte Carlo PDF simulation of supersonic combustion. Combust. Flame 132, 3-24 (2003)

15. Almeida, Y.P., Navarro-martinez, S.: Large Eddy Simulation of a supersonic lifted flame using the Eulerian stochastic fields method. Proc. Combust. Inst. 37, 3693-3701 (2019)

16. Ferrer, P.J.M.: Simulation Numérique Directe Dans La Combustion Turbulente Sur Une Couche De Cisaillement. Ph.D. Thesis, Ecole Nationale Supérieure de Mécanique et d'Aérotechique, Poitiers (2013)

17. Smagorinsky, J.: General circulation experiments with the primitive equations. Mon. Weather. Rev. 91(3), 99-164 (1963)

18. Villermaux, J., Devillon, J.C.: Représentation De La Coalescence Et De La Redispersion Des Domaines De SéGréGations Dans Un Fluide Par Un ModèLe D’interáction PhéNoméNologique. In: 2Nd International Symposium on Chemical Reaction Engineering. Netherlands (1972)

19. Jones, W.P., Marquis, A.J., Prasad, V.N.: LES of a turbulent premixed swirl burner using eulerian field method. Combust. Flame 159(10), 3079-3095 (2012)

20. Mustata, R., Valiño, L., Jiménez, C., Jones, W.P., Bondi, S.: A Probability Density function Eulerian Monte Carlo field method for large eddy simulations: Application to a turbulent piloted methane/air diffusion flame (Sandia D). Combust. Flame 145, 88-104 (2006)

21. Ribert, G., Petit, X., Domingo, P.: High-pressure methane-oxygen flames. Analysis of sub-grid scale contributions in filtered equations of state. J. Supercrit. Fluids 121, 78-88 (2017)

22. Azarnykh, D., Litvinov, S., Adams, N.A.: Numerical methods for the weakly compressible Generalized Langevin Model in Eulerian reference frame. J. Comput. Phys. 314, 93-106 (2016)

23. Petrova, N.: Turbulence-Chemistry Interaction Models for Numerical Simulation of Aeronautical Propulsion Systems. Ph.D. Thesis, Ecole Polytechnique, Paris (2015)

24. Nouri, A.G., Nik, M.B., Givi, P., Livescu, D., Pope, S.B.: Self-contained filtered density function. Phys. Rev. Fluids 2, 094603 (2017)

25. Yetter, R.A., Dryer, F.L., Rabitz, H.: A comprehensive reaction mechanism for carbon Monoxide/Hydrogen/Oxygen kinetics. Combust. Sci. Technol. 79, 97-128 (1991)

26. O'Conaire, M., Curran, H.J., Simmie, J.M., Pitz, W.J., Westbrook, C.K.: A Comprehensive Modeling Study of Hydrogen Oxidation. Int. J. Chem. Kinet. 36(11), 603-622 (2004)

27. Jones, W.P., Lyra, S., Navarro-Martinez, S.: Large eddy simulation of a swirl stabilized spray flame. Proc. Combust. Inst. 33, 2153-2160 (2011)

28. Jones, W.P., Navarro-Martinez, S.: Large eddy simulation of autoignition with a subgrid probability density function method. Combust. Flame 150, 170-187 (2007) 
29. Bell, J.H., Mehta, R.D.: Development of a two-stream mixing layer from tripped and untripped boundary layers. AIAA J. 28(12), 2034-2042 (1990)

30. Spencer, B.W., Jones, B.G.: Statistical investigation of pressure and velocity in the turbulent two-stream mixing layer. AIAA J. 71, 613-628 (1971)

31. Cheng, T.S., Wehrmeyer, J.A., Pitz, R.W., Jarrett, O. Jr., Northam, G.B.: Raman measurement of mixing and finite-rate chemistry in a supersonic hydrogen-air diffusion flame. Combust. Flame 99, 157-173 (1994)

Publisher's Note Springer Nature remains neutral with regard to jurisdictional claims in published maps and institutional affiliations. 